\title{
Somatic and Dendritic Small-Conductance Calcium- Activated Potassium Channels Regulate the Output of Cerebellar Purkinje Neurons
}

\author{
Mary D. Womack and Kamran Khodakhah \\ Department of Physiology and Biophysics, University of Colorado Health Sciences Center, Denver, Colorado 80262, and Department of Neuroscience, \\ Albert Einstein College of Medicine, Bronx, New York 10461
}

Cerebellar Purkinje neurons provide the sole output of the cerebellar cortex and play a crucial role in motor coordination and maintenance of balance. They are spontaneously active, and it is thought that they encode timing signals in the rate and pattern of their activity. An understanding of factors that control their excitability is important for delineating their computational role in the cerebellum. We evaluated the role of small-conductance calcium-activated potassium (SK) channels in the regulation of activity of mouse and rat Purkinje neurons. We find that somatic SK channels effectively limit the maximum firing rate of Purkinje neurons; when SK channels are blocked by the specific antagonists apamin or scyllatoxin, cells fire spontaneously at rates as high as 500 spikes per second. Dendritic SK channels, however, control primarily the extent to which dendrites contribute to the firing rate of Purkinje cells. Given their presence in the dendrites, it is likely that SK channels in the proximal dendrites govern the efficacy of dendrosomatic electrical coupling. When studied under physiological conditions, it is found that SK channels play the same role in controlling the excitability of adult Purkinje neurons as they do in young cells.

Key words: motor coordination; cerebellum; Purkinje cells; calcium-activated potassium channels; trimodal pattern of activity; apamin

\section{Introduction}

Purkinje neurons provide the sole output of the cerebellar cortex. It is believed that they encode timing information for motor coordination and maintenance of balance in their firing rate (Ito, 1984). Purkinje cells are intrinsically active in the absence of synaptic input (Hausser and Clark, 1997; Nam and Hockberger, 1997; Raman and Bean, 1997; Jaeger and Bower, 1999; Womack and Khodakhah, 2002). A great deal of evidence suggests that the intrinsic pattern and rate of activity of Purkinje cells are regulated developmentally (Woodward et al., 1969; Latham and Paul, 1971; Gruol et al., 1992; Falk et al., 1999). It was shown recently that, in the absence of fast excitatory and inhibitory synaptic inputs, in cerebellar slices young Purkinje cells fire predominantly at a regular tonic rate, whereas mature Purkinje cells have a trimodal pattern of activity whereby they cycle regularly between three phases, switching between tonic firing, bursting, and silent modes (Womack and Khodakhah, 2002). To understand the role of Purkinje cells in the cerebellar circuitry, it is important to delineate their input-output relationship. Clearly, such a task would be aided by an understanding of the intrinsic properties that affect their spontaneous activity and their excitability.

Small-conductance calcium-activated potassium (SK) channels are potassium channels that are activated by small changes in

\footnotetext{
Received Aug. 20, 2002; revised Jan. 13, 2003; accepted Jan. 15, 2003.

This project was supported by the Whitehall Foundation. We thank Dr. Paola Pedarzani for suggesting the experiments using EBIO and scyllatoxin, David Alevi and Felipe Castillo for gathering the data shown in Figures 1 and $6 \mathrm{~A}$ during their summer research in the laboratory, and Carolyn Chevez for technical support.

Correspondence should be addressed to Kamran Khodakhah, Department of Neuroscience, Albert Einstein College of Medicine, 506 Kennedy Center, 1410 Pelham Parkway South, Bronx, NY 10461. E-mail: kkhodakh@aecom.yu.edu.

Copyright $\odot 2003$ Society for Neuroscience $\quad 0270-6474 / 03 / 232600-08 \$ 15.00 / 0$
}

the cytosolic $\left[\mathrm{Ca}^{2+}\right]_{\mathrm{i}}$ (for review, see Vergara et al., 1998; Bond et al., 1999). In many neurons, they regulate the firing of the cell by contributing to a slow afterhyperpolarization (Sah, 1996) and by mediating spike frequency adaptation (Madison and Nicoll, 1984; Lancaster and Nicoll, 1987). There is evidence to suggest that these channels may play a crucial role in the cerebellum, because their block with apamin, a selective blocker of a class of SK channels, results in Purkinje cell atrophy (Mourre et al., 1997) and ataxia (Lallement et al., 1995). Furthermore, it was shown recently that rat Purkinje neurons express developmentally the apamin-sensitive SK2 but not SK1 and SK3 subunit mRNAs (Cingolani et al., 2002). The expression of SK2 channels is highest at early postnatal days and is reportedly downregulated within the first few weeks after birth (Cingolani et al., 2002).

We evaluated the role of apamin-sensitive SK channels in the pattern and rate of activity of mouse and rat Purkinje neurons. The role of these channels was investigated separately in cells with the trimodal and tonic patterns of activity. Furthermore, the function of dendritic and somatic SK channels in regulating firing was studied. We find that SK channels regulate the firing of both young and adult Purkinje neurons and that somatic and dendritic SK channels play somewhat different roles in this regulation. No differences were observed in the function of SK channels between the two species studied.

\section{Materials and Methods}

Preparation of slices. CD1 mice or Wistar rats at $10 \mathrm{~d}$ to $>3$ months after birth were anesthetized with halothane and killed by decapitation. Sagittal slices $300 \mu \mathrm{m}$ thick were prepared from the vermis of the cerebellum with a vibratome (Campden Instruments, Loughborough, UK). Slices were maintained at room temperature in the recording solution until use $(1-8 \mathrm{hr})$. 
Recording and analysis. Slices were mounted in a chamber on the stage of an upright Zeiss (Oberkochen, Germany) microscope and visualized using a $40 \times$ water immersion objective with infrared optics. Slices were superfused continuously at a rate of $1.5 \mathrm{ml} / \mathrm{min}$ with recording solution (in mM): $125 \mathrm{NaCl}, 2.5 \mathrm{KCl}, 26 \mathrm{NaHCO}_{3}, 1.25 \mathrm{NaH}_{2} \mathrm{PO}_{4}, 1 \mathrm{MgCl}_{2}, 2$ $\mathrm{CaCl}_{2}$, and 10 glucose, $\mathrm{pH} 7.4$ (when gassed with $5 \% \mathrm{CO}_{2}-95 \% \mathrm{O}_{2}$ ). Where indicated, the recording solution also contained kynurenic acid (5 $\mathrm{mm})$ and picrotoxin $(100 \mu \mathrm{M})$. The slice temperature was maintained at $35 \pm 0.5^{\circ} \mathrm{C}$ by adjusting the temperature of the bathing solution. The volume of the chamber was $\sim 2 \mathrm{ml}$, requiring several minutes for complete wash-in of the antagonists or blockers. For local perfusion, a glass pipette connected to a reservoir containing perfusate was positioned just above the surface of the slice. Fast green $(0.4 \%)$ or phenol red $(0.4 \%)$ was included in the perfusate to monitor the location of the perfusate. At these concentrations neither of the dyes produce a change in the firing of Purkinje neurons. A suction pipette was placed downstream from the perfusion pipette to limit the spread of the perfusate. The extent to which the perfusion was truly localized was assessed by monitoring the DC offset recorded by the differential amplifier when the perfusate was devoid of any ions (isotonic sucrose). Given our experimental setup and the position of perfusion and suction pipettes, the visible dye front was determined to be a reliable measure of the extent of localized perfusion.

Extracellular field potential recordings were made from individual Purkinje neurons using a home-made differential amplifier with glass pipette electrodes (tip size, $0.3-1 \mu \mathrm{m}$ ) filled with the recording solution. The pipette tip was positioned just above or lightly touching the cell body near the axon hillock, where the largest potential changes were usually recorded. Action potentials appeared as fast negative deflections of 50$1000 \mu \mathrm{V}$. Whole-cell recordings were made with borosilicate glass pipettes (3-6 M $\Omega$ ) filled with the following (in $\mathrm{mm}$ ): $140 \mathrm{~K}$-methyl sulfate, $10 \mathrm{KCl}, 5 \mathrm{NaCl}, 2 \mathrm{MgATP}, 0.01 \mathrm{EGTA}$, and 10 HEPES, pH 7.2. Perforated whole-cell recordings were made with similar pipettes filled with calcium-free recording solution to which $100 \mu \mathrm{M}$ glutamate and $10 \mu \mathrm{M}$ cyclothiazide were added. Whole-cell data were recorded using an Optopatch amplifier (Cairn Research, Faversham, UK). Data were sampled at $10 \mathrm{kHz}$ for extracellular recordings and at $20 \mathrm{kHz}$ for whole-cell recordings using a National Instruments (Austin, TX) analog-to-digital card (model MIO-16XE-10) and an IBM-compatible computer. Data acquisition and analyses were done with software written in-house using LabView (National Instruments). To analyze firing rate, a threshold level for spike detection was set by eye during the experiment. The number of spikes crossing the threshold was counted every $500 \mathrm{msec}$, and these are reported as the firing rate in terms of spikes per second. Data are reported as mean \pm SEM, and statistical significance was determined using oneway ANOVA. Kynurenic acid, picrotoxin, fast green, glutamate, cyclothiazide, and apamin were obtained from Sigma (St. Louis, MO). Methyl potassium sulfate was purchased from Pfaltz and Bauer (Waterbury, CT). Scyllatoxin was purchased from Latoxan (Valence, France). All other chemicals were of reagent grade.

\section{Results}

\section{Apamin increases the firing rate of Purkinje neurons}

To avoid alterations in the firing pattern of Purkinje cells, the activity of visually identified Purkinje cells in acutely prepared cerebellar slices was monitored with extracellular single-spike recordings at $35^{\circ} \mathrm{C}$. In the absence of pharmacological synaptic blockers, most mouse Purkinje cells fired tonically, whereas a few showed periods of bursting or silence (Womack and Khodakhah, 2002). Rat Purkinje neurons at 12-30 postnatal days also showed these two behaviors, as well as an additional type in which they cycled between periods of burst activity and silence (Fig. $1 A-C$ ). In all cases, bath perfusion of $100 \mathrm{~nm}$ apamin increased the firing rate dramatically (Fig. 1). The average predominant firing rates in the absence and presence of apamin were $41 \pm 4$ and $228 \pm 75$ spikes per second (mean $\pm \mathrm{SEM} ; n=5$ ), respectively, and the average maximum firing rate was $83 \pm 14$ and $520 \pm 33$ spikes per second (mean \pm SEM; $n=5$ ).
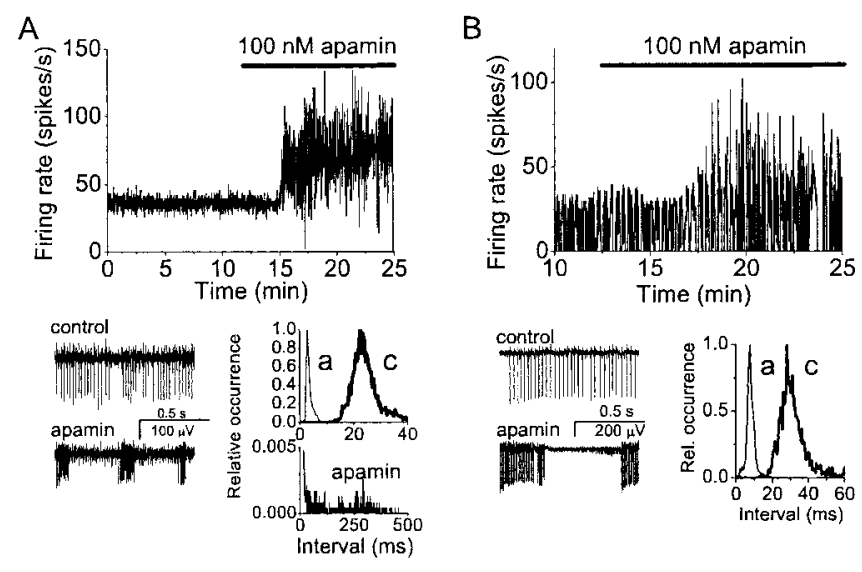

C
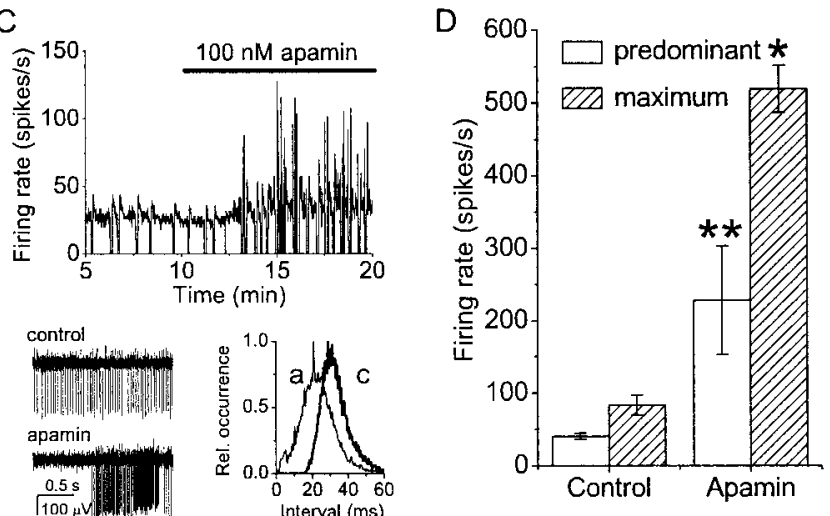

Figure 1. Block of SK channels increases the firing rate of Purkinje neurons. Activity of individual Purkinje neurons in rat cerebellar slices was recorded extracellularly using a differential amplifier. $A-C$, The records show the average firing rate and the effect of apamin. Samples of the extracellular records in the presence (top trace) and absence (bottom trace) of apamin are shown at bottom left of each panel. The relative occurrences of the interspike intervals are shown at the bottom right of each panel. A, Example of a tonic firing cell. Apamin made the cell burst at random and increased the firing rate. The lower interspike interval histogram shows the longer intervals corresponding to the intraburst intervals seen in the presence of apamin. Such long intervals were always seen when apamin was applied, but given their very low occurrence, they are omitted in the subsequent figures. $B$, Example of a cell that burst at random. Apamin exaggerated the bursts and increased the firing rate. $C$, Example of a cell with the trimodal pattern of activity. Apamin increased the firing rate and shortened the pattern period. $D$, The predominant and maximum firing rates were estimated from interspike histograms. The predominant firing rate is defined as the firing rate most often observed (the peak of the histogram). The maximum firing rate was defined as the fastest firing rate that was observed $5 \%$ of time relative to the predominant firing rate. The data reported are average values for five cells (mean \pm SEM). The mean values obtained in apamin were significantly different from those obtained under control conditions ( ${ }^{*} p<0.01,{ }^{* *} p<0.02$; determined by one-way ANOVA).

\section{Apamin does not increase the firing frequency of Purkinje} neurons by altering synaptic input

A possible interpretation of the results obtained above is that apamin affected the firing rate of Purkinje neurons by blocking SK channels of the excitatory and inhibitory neurons that make connections with Purkinje cells. To resolve this ambiguity, experiments were repeated under conditions in which the fast inhibitory and excitatory synaptic inputs were blocked by $100 \mu \mathrm{M}$ picrotoxin (Yoon et al., 1993) and $5 \mathrm{~mm}$ kyneurenic acid (Stone, 1993), respectively. In the presence of synaptic blockers, as reported previously (Womack and Khodakhah, 2002), Purkinje cells either fired tonically or showed a trimodal pattern of activity. In all tonic firing cells studied $(n=15)$, superfusion of apamin initially increased the firing rate and then made the cells fire in bursts (Fig. $2 A-D$ ). The increase in the predominant and maxi- 


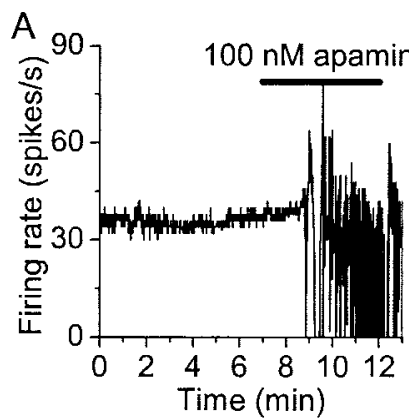

B

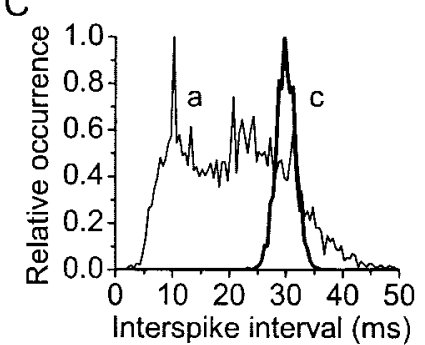

$\mathrm{D}$

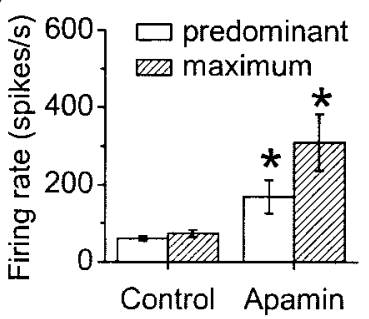

Figure 2. Block of SK channels increases the firing rate of tonically firing Purkinje neurons in the absence of synaptic input. $A$, Average firing rate from a neuron in a mouse cerebellar slice in the presence of fast excitatory and inhibitory synaptic blockers (5 mm kynurenate and $100 \mu \mathrm{m}$ picrotoxin). The tonic activity of the cells changed to random bursting when $100 \mathrm{~nm}$ apamin was bath applied. B, Sample extracellular records from the neuron described in $A$ in control solution (top trace) and in the presence of apamin (bottom trace). C, Relative distribution of interspike intervals for the cell described in $A$ under control conditions (c) and in apamin $(a)$. D, The average predominant and maximum firing rates for Purkinje neurons that fired tonically under control conditions (mean $\pm \mathrm{SEM}, n=7$ ). ${ }^{*}$ Statistical significance versus control conditions at $p<0.01$ determined by one-way ANOVA.

mum rates of firing was comparable with that seen in the absence of synaptic blockers (Fig. 2C,D).

The effect of blocking SK channels in the cells with the pattern was studied similarly. Bath perfusion of apamin also increased the firing rate (Fig. $3 A, C, D$ ) but did not disrupt the trimodal pattern of activity. The cell continued to cycle between the tonic, burst, and silent modes (Fig. $3 A, B$ ). Although the repetitive regular nature of the trimodal pattern of activity was left intact in apamin, the single-cycle duration was shortened significantly (Fig. $3 A, E$ ). This shortening was associated with a large reduction in the length of time that each cell spent in the tonic and burst modes without any appreciable change in the duration of the silent mode (Fig. $3 F$ ).

\section{SK channels contribute to afterhyperpolarizations after each action potential}

Although extracellular recording offers the possibility of monitoring the firing activity of Purkinje neurons without perturbing it, it provides little information regarding the absolute membrane potential during the activity. To test whether the membrane potential immediately after sodium spikes was affected by block of SK channels, a few experiments were performed in the currentclamp configuration of the patch-clamp technique. We used a potassium methyl sulfate internal solution because it has been shown to preserve SK currents. Gluconate-based solutions were avoided because gluconate has been suggested to block many ion channels, including potassium channels (Velumian et al., 1997) and, in Purkinje cells in slices, to result in the bursting of otherwise tonic firing neurons (T. Otis, personal communication). Figure $4 \mathrm{~A}$ shows the firing rate of a patch-clamped Purkinje neuron when the cell was allowed to fire spontaneously in the absence

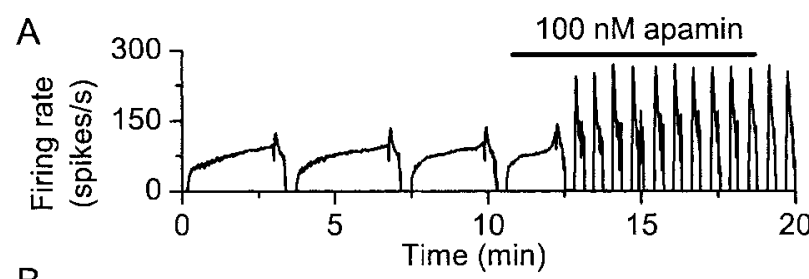

B
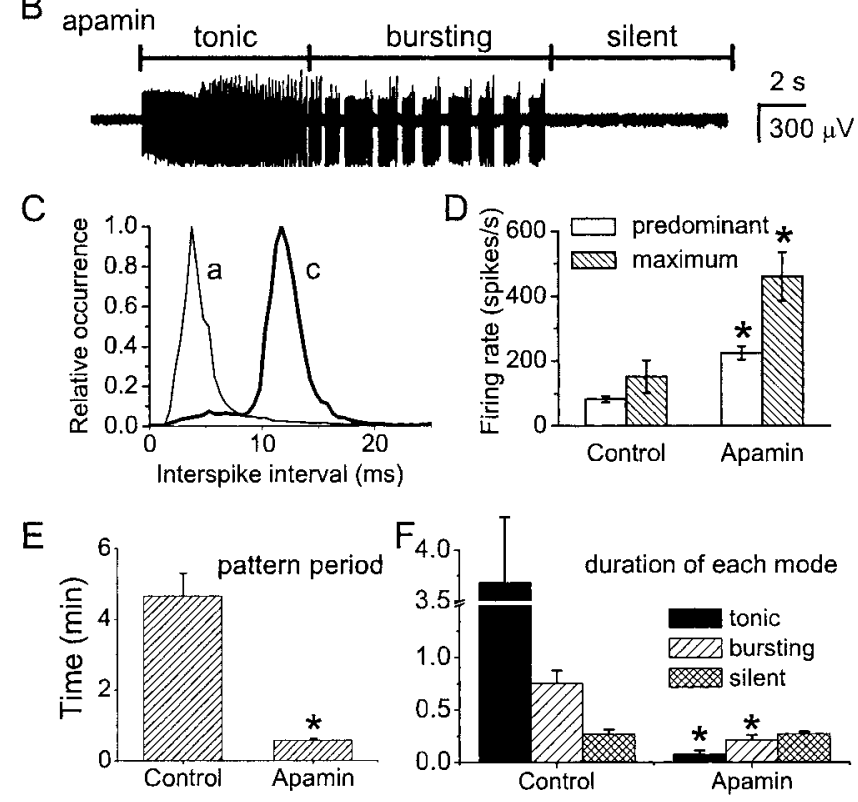

Figure 3. Block of SK channels increases the firing rate but maintains the trimodal pattern in cells that have the trimodal pattern of activity. $A$, Average firing rate from a neuron in a mouse cerebellar slice in the presence of synaptic blockers. In the absence of apamin, the cell exhibited a trimodal pattern of activity with a cycle duration of $\sim 5 \mathrm{~min}$. The cycle duration shortened and the firing rate increased in the presence of apamin. $B$, Sample extracellular records from the neuron described in $A$ in the presence of apamin. A single cycle of the trimodal pattern of activity is shown. A tonic firing phase is followed by periods of bursting. The interburst intervals gradually increase until the cell stops firing. After a period of silence, the pattern resumed with the start of another tonic firing phase. C, Distribution of interspike intervals for the cell described in $A$ under control conditions ( $c$ ) and in apamin $(a)$. D, The average predominant and maximum firing rates for Purkinje neurons that exhibited the trimodal pattern of activity (mean \pm SEM, $n=7$ ). *Statistical significance versus control conditions at $p<0.01$ determined by one-way ANOVA. $E$, The average duration of a single cycle of the trimodal pattern of activity (pattern period) in the presence and absence of $100 \mathrm{~nm}$ apamin (mean \pm SEM, $n=7$ ). *Statistical significance versus control conditions at $p<0.01$ by one-way ANOVA. F, Histograms show the average duration of the tonic phase of firing, the bursting phase, and the silent period in the presence and absence of apamin in cells with the trimodal pattern of activity (mean \pm SEM, $n=7) .{ }^{*}$ Statistical significance versus control conditions at $p<0.01$ determined by one-way ANOVA.

of any current injection. As apamin washed in, as seen with extracellular recordings, the firing rate increased until the cell started to burst (Fig. $4 B$ ). Figure $4 C$ shows averages of action potentials in the presence and absence of apamin. The membrane potential just before initiation of each action potential was not altered by block of the SK channels. There was also no change in the width of the action potential in the presence or absence of apamin. As the firing rate increased, however, the amplitude of the afterhyperpolarization decreased and the membrane potential depolarized more rapidly toward threshold. At $1 \mathrm{msec}$ after the peak of the action potential, apamin decreased the amplitude of the afterhyperpolarization by an average of $3.9 \pm 1 \mathrm{mV}$ (mean \pm SEM; $n=5$ ).

The shape of the average afterhyperpolarization was different during the bursts such that the immediate afterhyperpolarization 


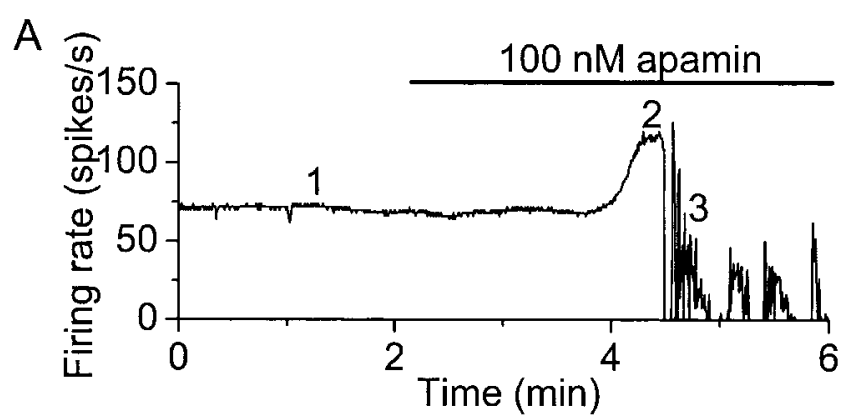

B

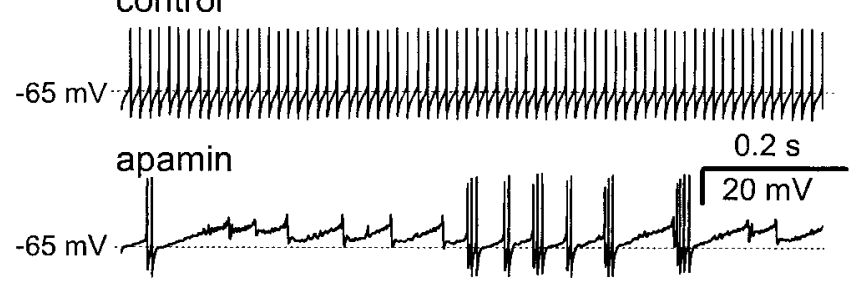

C

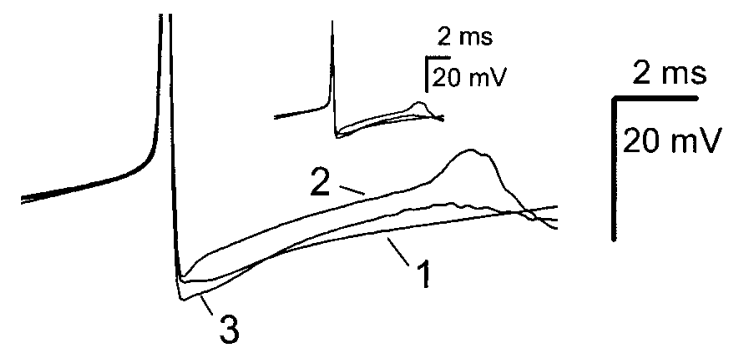

Figure 4. SK channels affect the action potential afterhyperpolarization in Purkinje neurons. A whole-cell current-clamp recording from a Purkinje neuron in a mouse cerebellar slice. $A$, Average firing rate of the current-clamped cell $(I=0)$ shows that apamin increased the firing rate and caused the cell to burst at random. $B$, Recordings of membrane potential from the neuron described in $A$ under control conditions (top trace) and in the presence of apamin (bottom trace). C, Average action potentials from the neuron described in $A$ recorded in control medium (1) and in the presence of apamin while the cell was firing tonically (2) or bursting (3). Each trace is the average of 30 seconds of firing. Numbers at the top of the trace in $A$ indicate the times at which action potentials were averaged. Action potentials have been truncated to show the afterhyperpolarization more clearly. The full action potentials are shown in the inset.

was larger than that under control conditions, but it then became smaller as the membrane potential depolarized rapidly (Fig. 4C). It is likely that the extent of contribution of various ion channels differs during burst firing compared with that during tonic firing. This differential contribution may account for the change in the shape and amplitude of the afterhyperpolarization seen during the bursts.

\section{The silent mode of the trimodal pattern of activity is not mediated by SK channels}

It has been speculated that the long silent mode of the trimodal pattern of activity is the consequence of membrane hyperpolarization, perhaps via calcium-dependent potassium channels (Womack and Khodakhah, 2002). However, there is no direct experimental evidence in support of the suggestion that calciumactivated potassium channels are responsible for the silent mode or that the silent mode is a consequence of hyperpolarization of the membrane potential rather than depolarization block. The main obstacle in obtaining these data has been the difficulty associated with recording the membrane potential with conventional whole-cell recordings while preserving the normal firing pattern of Purkinje cells. The traditional perforated recordings

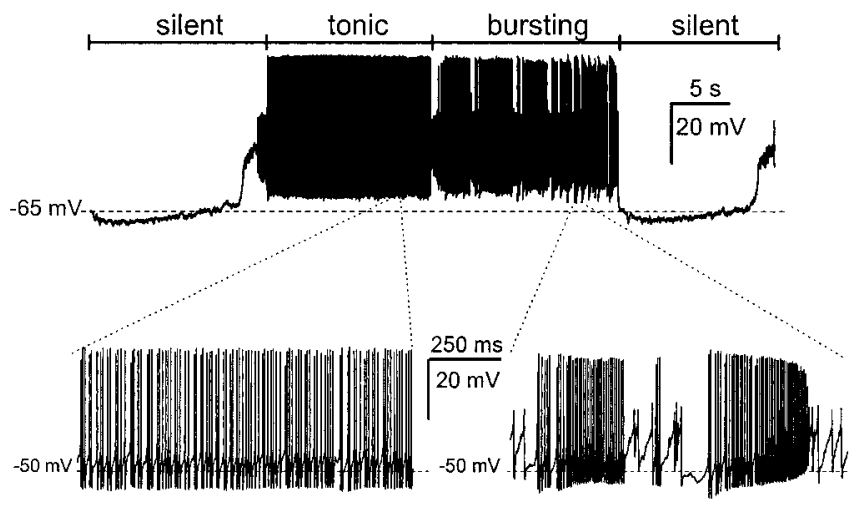

Figure 5. Silent periods during the trimodal pattern of activity are associated with long hyperpolarizations. The trimodal pattern of activity was recorded in the perforated whole-cell current-clamp configuration $(I=0)$ in the presence of apamin. Two silent hyperpolarized periods flank the tonic and bursting modes.

have proved to be impractical, given the long period of time required to capture a few cycles of the trimodal pattern of activity. We took advantage of the short duration of the trimodal pattern of activity in the presence of apamin to obtain perforated wholecell recordings in a few cells. By including glutamate and cyclothiazide in the patch pipette solution, we used the endogenous AMPA and kainate receptors (which in Purkinje cells have a reversal potential of $\sim 0 \mathrm{mV}$ ) to obtain electrical access to Purkinje cells. Cyclothiazide prevented desensitization of glutamateactivated AMPA receptors yielding an access resistance of 30-60 $\mathrm{M} \Omega$ during these whole-cell recordings. Although the relatively large access resistance combined with the pipette capacitance acted as a low-pass filter, resulting in an underestimation of fast signals, with the $I=0$ configuration of the patch-clamp amplifier (i.e., no current injection), there was no error in the slower changes in the membrane potential. This allowed us to examine the membrane potential during the silent periods of the trimodal pattern of activity. One such recording is shown in Figure 5. The tonic, burst, and silent modes of the trimodal pattern of activity can be identified clearly. The silent mode is associated with a prolonged hyperpolarization of the membrane potential to approximately $-65 \mathrm{mV}$, which in this cell was below threshold for activation of action potentials. These data demonstrate clearly that the silent mode of the pattern is the consequence of membrane hyperpolarization. Combined with the previous observation that apamin does not affect the duration of the silent mode (Fig. $3 F$ ), the results presented here suggest that, if the silent mode is the consequence of activation of calcium-activated potassium channels, these channels are not of the small-conductance type.

\section{SK channels regulate the firing of adult Purkinje neurons}

A recent report suggests that apamin-sensitive SK channels do not play a role in adult ( $60 \mathrm{~d}$ old) rat Purkinje cells (Cingolani et al., 2002). Because we had not noticed any reduction in the effectiveness of apamin in increasing the firing frequency of cells with the pattern, which were often from older animals (up to $30 \mathrm{~d}$ old), we revisited this question. We studied Purkinje cells in cerebella of the mothers of the pups used in the earlier sections, these animals being $\geq 3$ months old. Figure $6 A$ shows the firing rate of an adult rat Purkinje neuron in the absence of synaptic blockers. Block of SK channels with apamin increased the firing rate of the cell similarly to that seen in younger animals (Fig. 6A-C). The same increase in firing frequency was observed in adult mice, in the presence or the absence of synaptic blockers (Fig. 6D-F). 
A
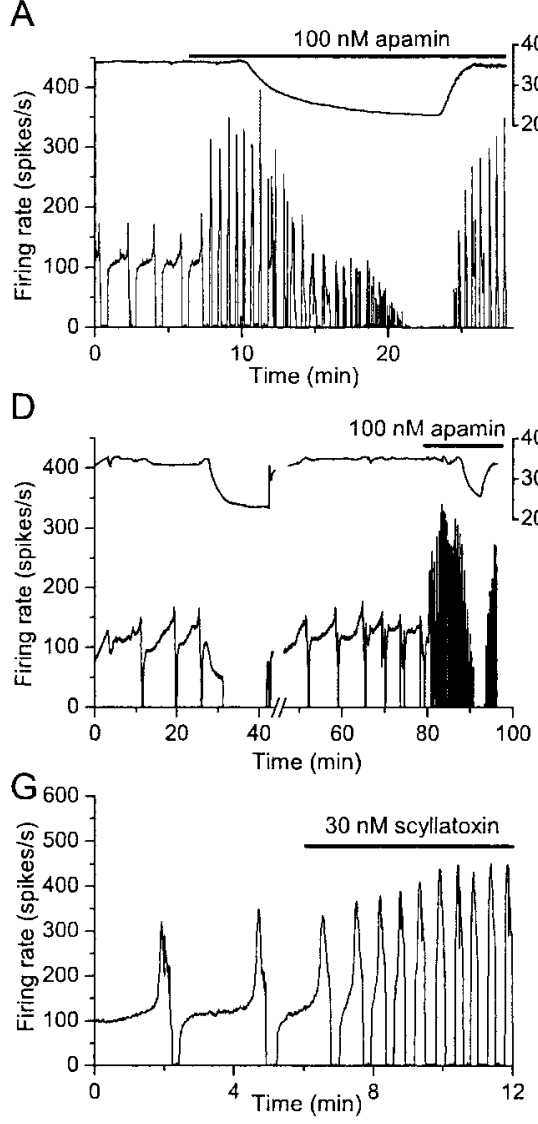

firing of Purkinje neurons was whether silent Purkinje cells that did not fire action potentials at rest displayed activity after addition of $100 \mathrm{~nm}$ apamin. The firing of Purkinje neurons is extremely sensitive to temperature, such that most cells with a trimodal pattern of activity do not show any activity at room temperature (Womack and Khodakhah, 2002). Because cells with the trimodal pattern of activity account for $>80 \%$ of adult Purkinje neurons, we examined whether, in the presence of apamin, Purkinje cells fired action potentials at room temperature. Figure $6 D$ shows one such experiment. The temperature dependence of firing of this adult Purkinje neuron was shown under control conditions. At temperatures $<27^{\circ} \mathrm{C}$, the cell was silent. At $35^{\circ} \mathrm{C}$, the cell showed the regular trimodal pattern of activity. Application of apamin increased the rate of activity of the cell significantly. When the temperature was decreased a second time in the presence of apamin, however, the cell again became quiescent. Qualitatively similar results were obtained in all cells tested (four each in mice and rats). This suggests that the inability of apamin to induce spontaneous activity in adult Purkinje neurons is a poor index of its functional role in these cells.

In cultured fetal heart cells, apamin has been shown to block $\mathrm{H}$

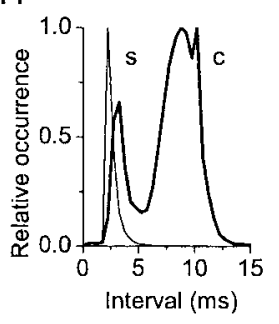

Figure 6. SK channels play an essential functional role in adult Purkinje neurons. Activity of individual Purkinje neurons was recorded using extracellular electrodes in cerebellar slices obtained from adult ( $>3$ months old) rats and mice. $A$, Firing rate of a Purkinje neuron in an adult rat cerebellar slice in the absence of synaptic blockers. The temperature of the solution bathing the slice is shown in the top trace. Apamin increased the firing rate and shortened the pattern duration. The cell stopped firing when the temperature was allowed to drop to room temperature in the presence of apamin. $B$, Relative occurrence of interspike intervals for the cell described in $A$ in control solution ( $c$ ) and in the presence of apamin ( $a$ ). C, Average of predominant (open bars) and maximum (hatched bars) firing rate for adult Purkinje neurons in the absence of synaptic blockers ( $n=4 ; 3$ rats and 1 mouse). ${ }^{*}$ Statistical significance versus control conditions at $p<0.01$ determined by one-way ANOVA. D, Firing rate of a Purkinje neuron in an adult mouse cerebellar slice in the presence of synaptic blockers. The cell stopped firing when the temperature was reduced to $27^{\circ} \mathrm{C}$ and resumed firing when it was increased back to $35^{\circ} \mathrm{C}$. Bath application of apamin increased the firing rate and reduced the pattern duration. Apamin did not prevent the cell from becoming quiescent when temperature was reduced a second time. $E$, Relative occurrence of interspike intervals for the cell described in $D$ in control solution $(c)$ and in the presence of apamin (a). F, Average of predominant (open bars) and maximum (hatched bars) firing rate for adult Purkinje neurons in the presence of synaptic blockers $(n=6 ; 3$ each of rats and mice). *Statistical significance versus control conditions at $p<0.01$ determined by one-way ANOVA. G, Firing rate of a Purkinje neuron in an adult rat cerebellar slice in the presence of synaptic blockers. Application of $30 \mathrm{~nm}$ scyllatoxin increased the firing rate and made the pattern period shorter. $H$, Relative occurrence of interspike intervals for the cell described in $G$ in control solution ( $c$ ) and in the presence of scyllatoxin ( $s$ ).

Experiments with adult animals were performed on a total of 12 cells with the same results (rats, $n=3$ without synaptic blockers, $n=4$ with synaptic blockers; mice, $n=1$ without synaptic blockers, $n=4$ with synaptic blockers). The predominant and maximum firing rates of Purkinje cells with apamin in adults were not significantly different from those in the young animals $(p>0.8$ and $p>0.1$ for predominant and maximum firing rates, respectively, determined by one-way ANOVA).

A major criterion used in the study by Cingolani et al. (2002) in assessing whether, at room temperature, apamin affected the

L-type calcium channels and TTX- and $\mathrm{Mn}^{2+}$-insensitive sodium currents (Bkaily et al., 1991, 1992). To ensure that the effects of apamin seen on adult Purkinje cells were not a result of its nonspecific effects, we repeated the experiments using the scorpion toxin scyllatoxin, a structurally different blocker of SK channels. As can be seen in Figure $6 G$, the effect of 30 nM scyllatoxin was not different from that of apamin. Scyllatoxin had similar effects in all four adult cells studied.

\section{SK channels are not saturated during the spontaneous firing of Purkinje cells}

We tested whether SK channels are saturated during spontaneous activity of Purkinje cells by superfusing the cells with 10 or 100 $\mu \mathrm{M}$ 1-ethyl-2-benzimidazolinone (EBIO), which shifts the apparent calcium affinity of SK channels to the lower nanomolar range (Pedarzani et al., 2001). It is expected that EBIO will not have an appreciable effect if SK channels are saturated. In tonic firing cells, application of EBIO reduced the firing rate significantly and reversibly (Fig. 7A, $B$ ) in all cells tested from $59 \pm 10$ to $18 \pm 7$ spikes per second ( \pm SEM; $n=9$ ). In the cells with the trimodal pattern of activity, EBIO also reduced the firing rate in all cells tested from $38 \pm 3$ to $12 \pm 1$ spikes per second $(n=5)$, and, in two of five cells, it changed their behavior reversibly, yielding tonic firing cells (Fig. $7 C, D$ ). The latter finding suggests that, depending on the conditions, the same Purkinje cell can either fire tonically or demonstrate the trimodal pattern of activity. The effect of EBIO on adult Purkinje cells was qualitatively the same as that on the young ones.

\section{Dendritic and somatic SK channels contribute differentially to spontaneous activity}

Purkinje cells have an extensive dendritic tree, which has been suggested to contribute actively to their spontaneous firing (Womack and Khodakhah, 2002). Using local perfusion of apamin, the extent to which dendritic and somatic SK channels affected the firing rate and pattern of activity of Purkinje neurons was investigated. An example of a local perfusion experiment on a tonic firing neuron is shown in Figure $8 \mathrm{~A}$. In this cell, perfusion of apamin on the outer half of the dendritic tree increased the firing rate and caused the cell to burst. Apamin was applied to the distal half of the dendritic tree in seven Purkinje cells. In three of the seven cells, an increase in the average firing rate was observed. 

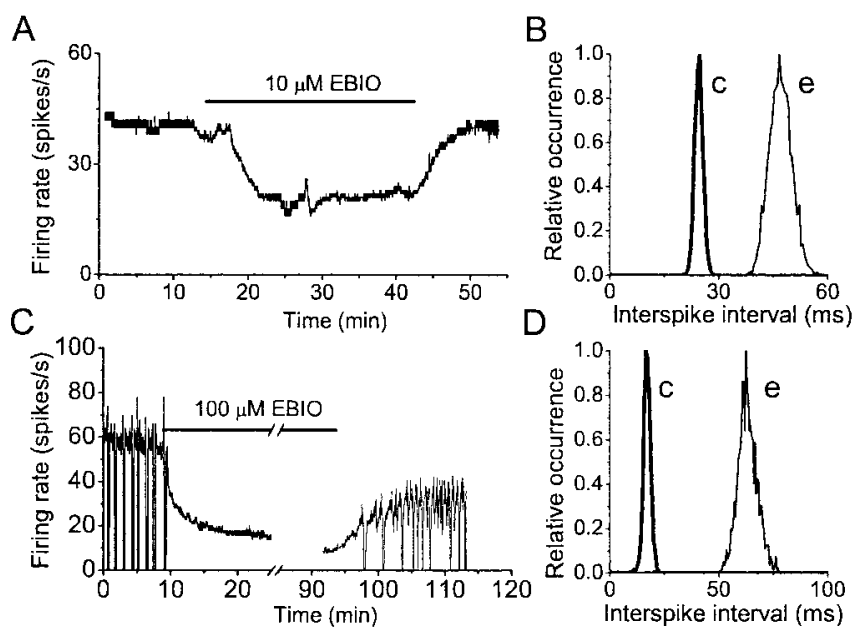

Figure 7. SK channels are not saturated during the spontaneous activity of Purkinje cells. $A$, Bath application of $10 \mu \mathrm{m} E B I 0$ to a tonic firing cell reversibly reduced the firing rate. $B$, Relative occurrence of interspike intervals for the cell described in $A$ under control conditions $(c)$ and in the presence of EBIO (e). Bath application of EBIO to a cell with the trimodal pattern of activity reduced the firing rate and made the cell fire tonically. The effects of EBIO were reversible. $D$, Relative occurrence of interspike intervals for the cell described in C under control conditions (c) or in EBIO (e).

In these three cells, the average firing rate, determined every 500 msec, increased from $72 \pm 9(n=3)$ to $125 \pm 30$ spikes per second ( \pm SEM; $n=3$ ). Apamin had no effect on the other four cells. When two-thirds of the dendritic tree was exposed to apamin, the average firing rate was higher in all four cells tested, increasing by $150 \pm 52 \%$ compared with the control firing rate (mean \pm SEM; $n=4)$. On occasion, and only in cells from older animals, when dendrites were exposed to apamin the cell assumed a rhythmic oscillatory pattern similar to the trimodal pattern of activity. Exposure of the soma to apamin further increased bursting and the rate of firing in all cells. The average firing rate when the entire cell was exposed to apamin, determined every $500 \mathrm{msec}$, increased by $260 \pm 13 \%$ of control levels (mean \pm SEM; $n=8$ ). Under these conditions, the predominant and maximum firing rates obtained from interspike histograms were the same as those seen when apamin was added to the bath perfusion.

Local perfusion experiments were also performed on 16 cells with the trimodal pattern of activity. Figure $8 B$ shows an experiment in which the outer two-thirds of the dendrites was exposed to apamin. In this cell, although the firing rate did not increase appreciably ( $20 \%$ increase vs control), the duration of the trimodal pattern was shortened significantly and disproportionally ( $90 \%$ reduction vs control). When apamin covered the soma in addition to the dendrites, firing rate increased by an additional $120 \%$, whereas the duration of the pattern decreased by only an additional 5\%. The firing pattern when the entire cell was perfused with apamin resembled that obtained with bath perfusion of apamin. Similar results were obtained in all 16 cells studied. Figure $8 C$ shows averages of the extent to which the pattern duration shortened and the firing rate increased as different proportions of dendrites were exposed to apamin in these experiments. As can be noted, selective blockade of dendritic SK channels had a disproportionate effect on the pattern period compared with the firing rate. In some experiments, such as the one shown in Figure $8 B$, even exposure of two-thirds of the dendrites to apamin did not increase the firing rate appreciably, yet it had a pronounced effect on the duration of the pattern.
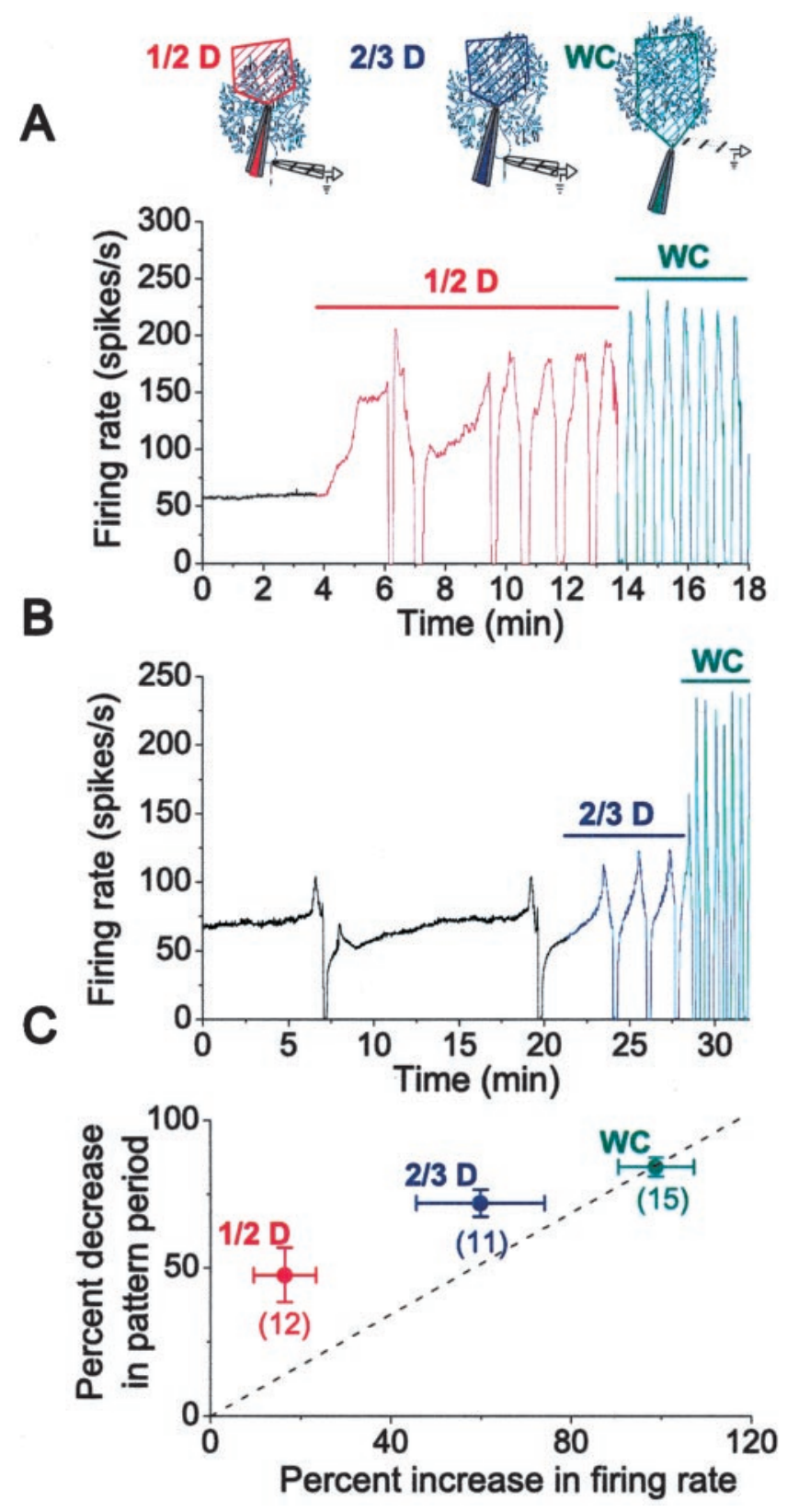

Figure8. DendriticSK channels contributeto spontaneous firing in Purkinjeneurons. Apamin (100 nM) was perfused locally onto the dendrites of mouse Purkinje neurons to assess the contribution of dendritic SK channels to spontaneous firing. Recordings were made in the presence of synaptic blockers. Diagrams at the top of each panel show the placement of the perfusion and recording pipettes relative to the Purkinjeneuron and the approximate area of the dendrites exposed to apamin. $A$, Firing rate of a Purkinje neuron that fired tonically in the absence of apamin (black trace). Apamin was applied selectively to the distal half $(1 / 2 D)$ of the dendritic tree (red trace) and then to the whole cell (WC) (green trace). $B$, Firing rate of a Purkinje neuron that exhibited a trimodal pattern of activity in the absence of apamin (black trace). Apamin was applied selectively to the distal two-thirds (2/3D) of the dendritic tree (blue trace) and then to the whole cell ( green trace).C, The average increase in firing rate induced by apamin in neurons with the trimodal pattern of activity is compared with the average decrease in the pattern period. Averages are shown for apamin applied locally to the distal half and distal two-thirds of the dendritic tree and to the whole cell. Results were obtained from neurons in the presence of synaptic blockers and are reported as mean \pm SEM. Numbers in the parentheses denote the number of experiments.

\section{Discussion}

Using single-cell extracellular spike recordings from Purkinje cells in acutely prepared rat or mouse cerebellar slices, we show that apamin-sensitive SK channels play a significant role in reg- 
ulating the spontaneous firing of Purkinje cells. As neurons mature, their intrinsic tonic firing behavior changes to a trimodal pattern of activity (Womack and Khodakhah, 2002). Although dendritic SK channels regulated firing of the both types of cells, in the cells with the trimodal pattern of activity, we find that dendritic SK channels contribute primarily to the single-cycle duration of the trimodal pattern, whereas the somatic channels limit the firing rate.

\section{SK channels regulate the firing of young and adult Purkinje neurons}

Purkinje cells express only the apamin-sensitive SK2 subunit of small-conductance calcium-activated potassium channels. In all experiments, block of SK channels with apamin or scyllatoxin increased the predominant and maximum firing frequencies by at least fivefold. The effect of apamin or scyllatoxin was mediated by blocking the SK channels present on Purkinje neurons rather than via a presynaptic mechanism, because it persisted in the presence of fast excitatory and inhibitory synaptic blockers.

The expression level of SK2 channels in rat Purkinje cells shows a marked decrease within the first few weeks after birth, at both the mRNA and protein levels, although it is important to note that some expression remains in the adult (Cingolani et al., 2002). The decrease in the expression level of SK channels has been reported to parallel a decrease in their regulatory role in Purkinje neurons, and it is proposed that these channels play little role in regulation of excitability of adult rat Purkinje neurons (Cingolani et al., 2002). In the present study, we find that, under physiological conditions, there is no difference in the functional significance of SK channels in regulating the firing rate of young or adult mouse or rat Purkinje neurons.

\section{Functional role of somatic SK channels}

Block of the somatic SK channels promoted bursting in Purkinje cells, in agreement with similar observations made in neostriatal cholinergic interneurons (Bennett et al., 2000), deep cerebellar neurons (Aizenman and Linden, 1999), and vestibular nucleus neurons (Smith et al., 2002). In addition, block of somatic SK channels increased the firing rate of Purkinje cells significantly, often permitting the cells to fire spontaneously in excess of 500 spikes per second as they burst. Thus, in Purkinje neurons, somatic SK channels efficiently prevent the cell from firing at extremely high rates.

In many neurons, SK channels contribute to a pronounced afterhyperpolarization mediated by the calcium entry during the action potential (Sah, 1996). Elimination of this afterhyperpolarization allows for the faster repolarization of the cell toward threshold and the earlier onset of a subsequent sodium spike. In Purkinje neurons, sodium spikes also increase somatic $\left[\mathrm{Ca}^{2+}\right]_{\mathrm{i}}$, which, in principle, can mediate an SK-dependent afterhyperpolarization. In current-clamp recordings, we find that, in the presence of apamin, the membrane potential depolarizes more quickly toward threshold after each action potential. Although this observation is in agreement with the suggestion that somatic SK channels contribute to the afterhyperpolarization, it is subject to alternative interpretation, because any manipulation that increases the firing rate implies that the membrane reaches threshold faster after each action potential. An alternative mechanism by which SK channels may regulate excitability of Purkinje cells is that they are tonically active at all times and thus reduce the membrane input resistance. In this case, block of SK channels will increase the input resistance, and a given inward current may depolarize the cell to such a great extent that the cell reaches threshold sooner. Given such a scenario, it might be expected that apamin should depolarize the cell, a phenomenon not observed here or in the study by Cingolani et al. (2002). In addition, because SK channels are not saturated by the bulk cytosolic $\left[\mathrm{Ca}^{2+}\right]_{\mathrm{i}}$, it would be difficult to imagine that the calcium influx mediated by each action potential does not result in an increase in the open probability of the SK channels and their contribution to the afterhyperpolarization after each action potential. To demonstrate unequivocally the presence of an SK channel-mediated afterhyperpolarization with each action potential, it would be necessary to measure the outward currents in voltage-clamped neurons in the presence and absence of apamin, because action potential waveforms are provided as command input. Given the difficulties in voltage clamping Purkinje cells because of their extensive dendritic tree, such a task is not practical.

\section{Functional role of dendritic SK channels}

The extensive dendritic tree of Purkinje cells has been suggested to contribute actively to their intrinsic firing (Cavelier et al., 2002; Womack and Khodakhah, 2002). The exact nature of the conductances that are involved in the dendritic electrical contribution to the soma is not well understood. In the tonic firing cells, we find that block of dendritic SK channels, sometimes solely in the outer half of the molecular layer, increases their firing rate significantly, often causing them to burst. Sodium action potentials do not invade far into the dendritic tree of Purkinje neurons (Llinas and Sugimori, 1980a,b; Stuart and Hausser, 1994), and calcium imaging experiments have shown that the extent of backpropagation of sodium spike-related calcium signals are limited to the initial proximal dendrites (K. Khodakhah, unpublished observations). Thus, it is unlikely that the dendritic SK channels are activated by calcium influx during each action potential and contribute to sodium spike afterhyperpolarization. Dendritic calcium spikes are also an unlikely source of calcium for dendritic SK channels in tonic firing Purkinje cells, because the regularity of sodium spike frequency in these cells, examined quantitatively by a study of their autocorrelograms and coefficient of variation of their interspike intervals (Womack and Khodakhah, 2002), argues against spontaneous dendritic calcium spikes. This supposition is supported by the finding that block of dendritic calcium channels by cadmium in the tonic firing cells does not affect their rate of activity (Womack and Khodakhah, 2002). Collectively, these suggest that, in the tonic firing Purkinje cells, the source of calcium for the dendritic SK channels is the resting dendritic $\left[\mathrm{Ca}^{2+}\right]_{i}$.

Functionally, block of the dendritic SK channels can affect activity of the cell by tightening the dendritic membrane and reducing dendritic leak and/or by allowing the dendrites to contribute a larger net inward current to the soma. The affinity of SK2 channels for calcium, the subtype present in Purkinje cells, is $\sim 0.3 \mu \mathrm{M}$ (Xia et al., 1998). Although an exact measurement of $\left[\mathrm{Ca}^{2+}\right]_{\mathrm{i}}$ in the dendrites of Purkinje neurons is not available, it is unlikely that, in the absence of calcium spikes, it is high enough to saturate the dendritic SK channels. This suggests that dendritic SK channels can take a more active role in regulation of the dendritic excitability when calcium is elevated, such as when the cell receives an input from its attendant climbing fiber. In addition, because action potential-related calcium signals backpropagate into the proximal dendrites (K. Khodakhah, unpublished observations) and the extent of the calcium rise is proportional to the firing rate, it is likely that the extent of activation of SK channels in this region is also critically dependent on the firing frequency 
of the cell. This suggests that SK channels may modulate the efficiency of the electrical coupling of the dendrites to the soma by making the proximal dendrites more leaky to potassium ions when $\left[\mathrm{Ca}^{2+}\right]_{\mathrm{i}}$ is elevated. Under these conditions, the coupling efficiency would be inversely proportional to the rate of activity of the cell, lessening the impact of synaptic inputs at higher frequencies.

We also tested the role of dendritic SK channels in the more mature Purkinje cells with the trimodal pattern of activity. Intriguingly, in many instances, block of distal SK channels did not alter the firing rate significantly but shortened detectably the duration of the trimodal pattern. In cells with the trimodal pattern of activity, dendritic calcium channels make a significant contribution to the firing pattern, because their block with cadmium causes the cells to burst and halts firing (Womack and Khodakhah, 2002). It is also likely that the bursts of sodium spikes during the burst mode of the pattern are associated with calcium spikes in the dendrites. Blockade of the dendritic SK channels shortens the period of the trimodal pattern, suggesting that under these conditions, the dendrites can initiate calcium spikes more readily. Dendritic SK channels in the proximal dendrites of cells with the pattern are also likely to play the same role in controlling the electrical coupling efficiency between the soma and dendrites as discussed for the tonic firing neurons.

The data presented here show that SK channels regulate the excitability of cerebellar Purkinje neurons. Their presence in the dendrites allows for not only modulation of the spontaneous activity of the cell but also control of the efficiency by which dendritic inputs are relayed to the soma.

\section{References}

Aizenman CD, Linden DJ (1999) Regulation of the rebound depolarization and spontaneous firing patterns of deep nuclear neurons in slices of rat cerebellum. J Neurophysiol 82:1697-1709.

Bennett BD, Callaway JC, Wilson CJ (2000) Intrinsic membrane properties underlying spontaneous tonic firing in neostriatal cholinergic interneurons. J Neurosci 20:8493-8503.

Bkaily G, Jacques D, Sculptoreanu A, Yamamoto T, Carrier D, Vigneault D, Sperelakis N (1991) Apamin, a highly potent blocker of the TTX- and $\mathrm{Mn}^{2+}$-insensitive fast transient $\mathrm{Na}^{+}$current in young embryonic heart. J Mol Cell Cardiol 23:25-39.

Bkaily G, Sculptoreanu A, Jacques D, Economos D, Menard D (1992) Apamin, a highly potent fetal L-type $\mathrm{Ca}^{2+}$ current blocker in single heart cells. Am J Physiol 262:H463-H471.

Bond CT, Maylie J, Adelman JP (1999) Small-conductance calciumactivated potassium channels. Ann NY Acad Sci 868:370-378.

Cavelier P, Pouille F, Desplantez T, Beekenkamp H, Bossu JL (2002) Control of the propagation of dendritic low-threshold $\mathrm{Ca}^{2+}$ spikes in Purkinje cells from rat cerebellar slice cultures. J Physiol (Lond) 540:57-72.

Cingolani LA, Gymnopoulos M, Boccaccio A, Stocker M, Pedarzani P (2002) Developmental regulation of small-conductance $\mathrm{Ca}^{2+}$-activated $\mathrm{K}^{+}$ channel expression and function in rat Purkinje neurons. J Neurosci 22:4456-4467.

Falk T, Muller YL, Yool AJ (1999) Differential expression of three classes of voltage-gated $\mathrm{Ca}^{2+}$ channels during maturation of the rat cerebellum in vitro. Brain Res Dev Brain Res 115:161-170.

Gruol DL, Deal CR, Yool AJ (1992) Developmental changes in calcium conductances contribute to the physiological maturation of cerebellar Purkinje neurons in culture. J Neurosci 12:2838-2848.

Hausser M, Clark BA (1997) Tonic synaptic inhibition modulates neuronal output pattern and spatiotemporal synaptic integration. Neuron 19: 665-678.

Ito M (1984) The cerebellum and neural control. New York: Raven.

Jaeger D, Bower JM (1999) Synaptic control of spiking in cerebellar Purkinje cells: dynamic current clamp based on model conductances. J Neurosci 19:6090-6101.

Lallement G, Fosbraey P, Baille-Le-Crom V, Tattersall JE, Blanchet G, Wetherell JR, Rice P, Passingham SL, Sentenac-Roumanou H (1995) Compared toxicity of the potassium channel blockers, apamin and dendrotoxin. Toxicology 104:47-52.

Lancaster B, Nicoll RA (1987) Properties of two calcium-activated hyperpolarizations in rat hippocampal neurones. J Physiol (Lond) 389:187-203.

Latham A, Paul DH (1971) Spontaneous activity of cerebellar Purkinje cells and their responses to impulses in climbing fibres. J Physiol (Lond) 213:135-156.

Llinas R, Sugimori M (1980a) Electrophysiological properties of in vitro Purkinje cell somata in mammalian cerebellar slices. J Physiol (Lond) 305:171-195.

Llinas R, Sugimori M (1980b) Electrophysiological properties of in vitro Purkinje cell dendrites in mammalian cerebellar slices. J Physiol (Lond) 305:197-213.

Madison DV, Nicoll RA (1984) Control of the repetitive discharge of rat CA 1 pyramidal neurones in vitro. J Physiol (Lond) 354:319-331.

Mourre C, Fournier C, Soumireu-Mourat B (1997) Apamin, a blocker of the calcium-activated potassium channel, induces neurodegeneration of Purkinje cells exclusively. Brain Res 778:405-408.

Nam SC, Hockberger PE (1997) Analysis of spontaneous electrical activity in cerebellar Purkinje cells acutely isolated from postnatal rats. J Neurobiol 33:18-32.

Pedarzani P, Mosbacher J, Rivard A, Cingolani LA, Oliver D, Stocker M, Adelman JP, Fakler B (2001) Control of electrical activity in central neurons by modulating the gating of small conductance $\mathrm{Ca}^{2+}$-activated $\mathrm{K}^{+}$ channels. J Biol Chem 276:9762-9769.

Raman IM, Bean BP (1997) Resurgent sodium current and action potential formation in dissociated cerebellar Purkinje neurons. J Neurosci 17:4517-4526.

Sah P (1996) $\mathrm{Ca}^{2+}$-activated $\mathrm{K}^{+}$currents in neurones: types, physiological roles and modulation. Trends Neurosci 19:150-154.

Smith MR, Nelson AB, Du LS (2002) Regulation of firing response gain by calcium-dependent mechanisms in vestibular nucleus neurons. J Neurophysiol 87:2031-2042.

Stone TW (1993) Neuropharmacology of quinolinic and kynurenic acids. Pharmacol Rev 45:309-379.

Stuart G, Hausser M (1994) Initiation and spread of sodium action potentials in cerebellar Purkinje cells. Neuron 13:703-712.

Velumian AA, Zhang L, Pennefather P, Carlen PL (1997) Reversible inhibition of IK, IAHP, Ih and ICa currents by internally applied gluconate in rat hippocampal pyramidal neurones. Pflügers Arch 433:343-350.

Vergara C, Latorre R, Marrion NV, Adelman JP (1998) Calcium-activated potassium channels. Curr Opin Neurobiol 8:321-329.

Womack M, Khodakhah K (2002) Active contribution of dendrites to the tonic and trimodal patterns of activity in cerebellar Purkinje neurons. J Neurosci 22:10603-10612.

Woodward DJ, Hoffer BJ, Lapham LW (1969) Postnatal development of electrical and enzyme histochemical activity in Purkinje cells. Exp Neurol 23:120-139.

Xia XM, Fakler B, Rivard A, Wayman G, Johnson-Pais T, Keen JE, Ishii T, Hirschberg B, Bond CT, Lutsenko S, Maylie J, Adelman JP (1998) Mechanism of calcium gating in small-conductance calcium-activated potassium channels. Nature 395:503-507.

Yoon KW, Covey DF, Rothman SM (1993) Multiple mechanisms of picrotoxin block of GABA-induced currents in rat hippocampal neurons. J Physiol (Lond) 464:423-439. 